\title{
Combining data mining algorithm and object-based image analysis for detailed urban mapping of hyperspectral images
}

\begin{abstract}
Image classification of roofing types, road pavements, and natural features can assist landcover maps in further examining the effects of such features on health, pollution, and the microclimate in urban settings. Airborne hyperspectral sensors with high spectral and spatial resolutions can be employed for detailed characterization of urban areas. This study aims to develop a procedure that is instrumental for automated knowledge discovery and mapping of urban surface materials from a large feature space of hyperspectral images. Two different images over Universiti Putra Malaysia (UPM) and Kuala Lumpur (KL), Malaysia, were captured by using hyperspectral sensors with 20 and 128 bands. The images were used to explore the combined performance of a data mining (DM) algorithm and object-based image analysis (OBIA). A large number of attributes were discovered with the C4.5 DM algorithm, which also generated the classification model as a decision tree. The UPM and KL classified images achieved 93.42 and $88.36 \%$ overall accuracy. The high accuracy of object-based classification can be linked to the knowledge discovery produced by the DM algorithm. This algorithm increased the productivity of OBIA, expedited the process of attribute selection, and resulted in an easy-to-use representation of a knowledge model from a decision tree structure.
\end{abstract}

Keyword: Data mining (DM) algorithm; Object-based image analysis (OBIA); Urban mapping; Hyperspectral images 